\title{
Stability of solitonic solutions of Super KdV equations under susy breaking conditions
}

\author{
Alvaro Restuccia ${ }^{1,2}$ and Adriań Sotomayor ${ }^{3 *}$
}

\section{"Correspondence:}

asotomayor@uantof.cl

${ }^{3}$ Departamento de Matemáticas,

Universidad de Antofagasta,

Antofagasta, Chile

Full list of author information is

available at the end of the article

\begin{abstract}
A supersymmetric breaking procedure for $N=1$ Super Korteweg-de Vries (SKdV), preserving the positivity of the hamiltonian as well as the existence of solitonic solutions, is implemented. The resulting solitonic system is shown to have nice stability properties.
\end{abstract}

PACS Codes: $12.60 . J v ; 02.30 .1 k ; 11.30 .-j ; 05.45 . Y v ; 02.30 . J r$

Keywords: supersymmetric models; integrable systems; conservation laws; nonlinear dynamics of solitons; partial differential equations

\section{Introduction}

The breaking of supersymmetry in physical systems is always an interesting aspect to analyze. We consider a solitonic system [1] arising from the breaking of supersymmetry in the $N=1$ Super KdV system [2,3]. In the latter, there is only one hamiltonian structure in distinction to the bi-hamiltonian one in the Korteweg-de Vries (KdV) system. The $N=1$ SKdV hamiltonian, however, is not manifestly positive. Nevertheless, the quantum formulation of the theory yields a manifestly positive definite self-adjoint operator. The stability of the ground state of the system is then assured from it. We considered in [1] the supersymmetry breaking of the SKdV system by changing the Grassmann algebra structure of the SKdV formulation to a Clifford algebra one. This susy breaking mechanism has already appeared in several works, see for example [4]. One may then obtain a solitonic system with the same evolution equation for the new Clifford algebra-valued field as one had for the odd Grassmann algebra-valued one in the SKdV system, and what is more important, with a bounded from below hamiltonian. The system presents solitonic solutions, although the infinite set of local conserved quantities of SKdV breaks down. We will show in this work that this solitonic system has nice stability properties.

The stability in the sense of Liapunov of the one-soliton solution of KdV equation was first proven by Benjamin [5] and Bona [6]. The proof makes use of the first few conserved quantities of the $\mathrm{KdV}$ equation. In particular, the fact that one of them is the square of $L_{2}$ norm is relevant in their argument. The use of conserved quantity was also considered in a stability argument by Boussinesq [7]. In this paper, we make use of this main idea to prove stability of the one-soliton solution of the coupled equation, with fields valued on a Clifford algebra, derived from the supersymmetric breaking of the $N=1 \mathrm{SKdV}$ equation. 


\section{$2 \mathrm{SKdV}$ and the breaking of supersymmetry}

The fields $u(x, t)$ and $\xi(x, t)$ describing $N=1 \mathrm{SKdV}$ equations [2] take values on the even and odd part of a Grassmann algebra, respectively. The $N=1 \mathrm{SKdV}$ equations are

$$
\begin{aligned}
& u_{t}=-u^{\prime \prime \prime}+6 u u^{\prime}-3 \xi \xi^{\prime \prime}, \\
& \xi_{t}=-\xi^{\prime \prime \prime}+3(\xi u)^{\prime} .
\end{aligned}
$$

This system of partial differential equations has infinite local conserved charges as well as infinite non-local conserved charges [2,8-11]. The first few local ones are

$$
\begin{aligned}
& H_{\frac{1}{2}}=\int_{-\infty}^{+\infty} \xi d x, \quad H_{1}=\int_{-\infty}^{+\infty} u d x, \\
& H_{3}=\int_{-\infty}^{+\infty}\left(u^{2}-\xi \xi^{\prime}\right) d x, \quad H_{5}=\int_{-\infty}^{+\infty}\left(2 u^{3}+\left(u^{\prime}\right)^{2}-\xi^{\prime} \xi^{\prime \prime}-4 u \xi \xi^{\prime}\right) d x .
\end{aligned}
$$

To break supersymmetry, we consider the fields $u$ and $\xi$ taking values on a Clifford algebra instead of being Grassmann algebra-valued. We thus take $u$ to be a real-valued field, while $\xi$ to be an expansion in terms of the generators $e_{i}, i=1, \ldots$ of the Clifford algebra:

$$
\xi=\sum_{i} \varphi_{i} e_{i}+\sum_{i j} \varphi_{i j} e_{i} e_{j}+\sum_{i j k} \varphi_{i j k} e_{i} e_{j} e_{k}+\cdots
$$

where

$$
e_{i} e_{j}+e_{j} e_{i}=-2 \delta_{i j}
$$

and $\varphi_{i}, \varphi_{i j}, \varphi_{i j k}, \ldots$ are real-valued functions. We define $\bar{\xi}=\sum_{i=1}^{\infty} \varphi_{i} \bar{e}_{i}+\sum_{i j} \varphi_{i j} \bar{e}_{j} \bar{e}_{i}+\sum_{i j k} \varphi_{i j k} \times$ $\bar{e}_{k} \bar{e}_{j} \bar{e}_{i}+\cdots$, where $\bar{e}_{i}=-e_{i}$. We denote as in superfield notation the body of the expansion those terms associated with the identity generator and the soul the remaining ones. Consequently, the body of $\xi \bar{\xi}$, denoted by $\mathcal{P}(\xi \bar{\xi})$, is equal to $\sum_{i} \varphi_{i}^{2}+\sum_{i j} \varphi_{i j}^{2}+\sum_{i j k} \varphi_{i j k}^{2}+\cdots$. In what follows, without loss of generality, we rewrite $\mathcal{P}(\xi \bar{\xi})=\sum_{i} \varphi_{i}^{2}+\sum_{i j} \varphi_{i j}^{2}+\sum_{i j k} \varphi_{i j k}^{2}+\cdots$ simply as $\mathcal{P}(\xi \bar{\xi})=\sum_{i} \varphi_{i}^{2}$.

The system of partial differential equations arising from the breaking of supersymmetry, which has the required properties as discussed in [1] is

$$
\begin{aligned}
& u_{t}=-u^{\prime \prime \prime}-u u^{\prime}-\frac{1}{4}(\mathcal{P}(\xi \bar{\xi}))^{\prime}, \\
& \xi_{t}=-\xi^{\prime \prime \prime}-\frac{1}{2}(\xi u)^{\prime} .
\end{aligned}
$$

System (5) has the following conserved charges:

$$
\begin{aligned}
& \hat{H}_{\frac{1}{2}}=\int_{-\infty}^{+\infty} \xi d x \\
& \hat{H}_{1}=\int_{-\infty}^{+\infty} u d x \\
& V \equiv \hat{H}_{3}=\int_{-\infty}^{+\infty}\left(u^{2}+\mathcal{P}(\xi \bar{\xi})\right) d x \\
& M=\hat{H}_{5}=\int_{-\infty}^{+\infty}\left(-\frac{1}{3} u^{3}-\frac{1}{2} u \mathcal{P}(\xi \bar{\xi})+\left(u^{\prime}\right)^{2}+\mathcal{P}\left(\xi^{\prime} \bar{\xi}^{\prime}\right)\right) d x .
\end{aligned}
$$


The system has multi-solitonic solutions, for example, $u(x, t) \equiv \phi(x, t)=3 \mathcal{C} \frac{1}{\cosh ^{2}(z)}, z \equiv$ $\frac{1}{2} \mathcal{C}^{\frac{1}{2}}(x-(1+\mathcal{C}) t+a), \xi(x, t)=0$, where $\phi(x, t)$ is the one-soliton solution of $\mathrm{KdV}$ equation, $a$ is an arbitrary real number, and $\mathcal{C}>0$.

This system with a change of sign in the third term of the right-hand member of the first equation of system (5) was considered in $[12,13]$.

It will be important in the following stability argument that

$$
\int_{-\infty}^{\infty} \mathcal{P}(\xi \bar{\xi}) d x=\|\xi\|_{L_{2}}^{2}
$$

hence $V$ is $L_{2}$ norm of $(u, \xi)$. This property is absent for the system with a positive sign on the third term of the first equation in (5).

\section{Stability properties of the system}

We may now analyze the stability of the ground state as well as the stability of the onesoliton solution of system (5).

We start the analysis by considering an a priori bound for the solutions of system (5). We denote by \|\|$_{H_{1}}$ the Sobolev norm

$$
\|(u, \xi)\|_{H_{1}}^{2}=\int_{-\infty}^{+\infty}\left[\left(u^{2}+\sum_{i=0}^{\infty} \varphi_{i}^{2}\right)+\left({u^{\prime 2}}^{2}+\sum_{i=0}^{\infty} \varphi_{i}^{\prime 2}\right)\right] d x
$$

We obtain

$$
\|(u, \xi)\|_{H_{1}}^{2} \leq V+M+\frac{1}{2} \int_{-\infty}^{+\infty}|u|\left(u^{2}+\mathcal{P}(\xi \bar{\xi})\right) d x
$$

we now use

$$
\sup |u| \leq \frac{1}{\sqrt{2}}\|u\|_{H_{1}} \leq \frac{1}{\sqrt{2}}\|(u, \xi)\|_{H_{1}}
$$

it yields

$$
\|(u, \xi)\|_{H_{1}}^{2} \leq V+M+\frac{1}{2 \sqrt{2}} V\|(u, \xi)\|_{H_{1}} .
$$

From (11), it follows that

$$
\|(u, \xi)\|_{H_{1}} \leq \frac{d+\sqrt{d^{2}+4 e}}{2}
$$

where $d=\frac{1}{2 \sqrt{2}} V$ and $e=V+M$. We notice that $d^{2}+4 e \geq 0$.

Consequently, given $V$ and $M$, from the initial data and a solution satisfying those initial conditions, the $\|(u, \xi)\|_{H_{1}}$ is bounded by (12). The a priori bound is a strong evidence of the existence of the solution for $0 \leq t$. We will consider this existence problem elsewhere. In this work, we assume the existence of the solution and its continuous dependence on the initial data under smooth enough assumptions on the initial perturbation. 
We consider the stability in the sense of Liapunov. In particular, we take the same definition as in [5]: $(\hat{u}, \hat{\xi})$, a solution of (5), is stable if given $\epsilon$, there exists $\delta$ such that for any solution $(u, \xi)$ of (5), satisfying at $t=0$

$$
d_{I}[(u, \xi),(\hat{u}, \hat{\xi})]<\delta
$$

then

$$
d_{I I}[(u, \xi),(\hat{u}, \hat{\xi})]<\epsilon
$$

for all $t \geq 0$.

$d_{I}$ and $d_{I I}$ denote two distances to be defined.

We consider now the stability problem of the ground state solution $\hat{u}=0, \hat{\xi}=0$.

We take $d_{I}$ and $d_{I I}$ to be the Sobolev norm $\|(u-\hat{u}, \xi-\hat{\xi})\|_{H_{1}}$.

We get

$$
V \leq\|(u, \xi)\|_{H_{1}}^{2}
$$

and

$$
M \leq \int_{-\infty}^{+\infty}\left(\frac{1}{2}|u|\left(u^{2}+\mathcal{P}(\xi \bar{\xi})\right)+u^{\prime 2}+\mathcal{P}\left(\xi^{\prime} \bar{\xi}^{\prime}\right)\right) d x \leq \frac{1}{2 \sqrt{2}}\|(u, \xi)\|_{H_{1}}^{3}+\|(u, \xi)\|_{H_{1}}^{2} .
$$

At $t=0$, we then have, using (13),

$$
\begin{aligned}
& V<\delta^{2}, \\
& M<\frac{1}{2 \sqrt{2}} \delta^{3}+\delta^{2} .
\end{aligned}
$$

Consequently, from the a priori bound (12), we obtain for any $t \geq 0$

$$
\|(u, \xi)\|_{H_{1}}<\epsilon
$$

for any given $\epsilon$, provided $\delta$ is conveniently chosen.

This argument proves the stability of the ground state solution.

We now consider the stability of the one-soliton solution $\hat{u}=\phi, \hat{\xi}=0$. The proof of stability is based on estimates for the $u$ field, which are analogous to the one presented in $[5,6]$ while a new argument will be given for the $\xi$ field. The distances we will use are

$$
\begin{aligned}
& d_{I}\left[\left(u_{1}, \xi_{1}\right),\left(u_{2}, \xi_{2}\right)\right]=\left\|\left(u_{1}-u_{2}, \xi_{1}-\xi_{2}\right)\right\|_{H_{1}}, \\
& d_{I I}\left[\left(u_{1}, \xi_{1}\right),\left(u_{2}, \xi_{2}\right)\right]=\inf _{\tau}\left\|\left(\tau u_{1}-u_{2}, \xi_{1}-\xi_{2}\right)\right\|_{H_{1}},
\end{aligned}
$$

where $\tau u_{1}$ denotes the group of translations along the $x$-axis. $d_{I I}$ is a distance on a metric space obtained by identifying the translations of each $u \in H_{1}(\mathbb{R})[5] . d_{I I}$ is related to a stability in the sense that a solution $u$ remains close to $\hat{u}=\phi$ only in shape but not necessarily in position. 
We first assume that

$$
V(u, \xi)=V(\hat{u}, \hat{\xi})=V(\phi, 0)
$$

and

$$
\int_{-\infty}^{+\infty} \xi d x=\int_{-\infty}^{+\infty} \hat{\xi} d x=0
$$

and then we will relax this conditions to get the most general formulation of the stability problem.

Following [5], we define

$$
h(x, t)=u(x-a, t)-\phi(x+\mathcal{C} t)
$$

where $a$ is defined, for each $t \geq 0$, by

$$
\int_{-\infty}^{+\infty}[u(x, t)-\phi(x+a)]^{2} d x=\inf _{y \in \mathbb{R}} \int_{-\infty}^{+\infty}[u(x, t)-\phi(x+y)]^{2} d x .
$$

In [6], it was proven that the infimum is taken on finite values of $y$.

We obtain

$$
\begin{aligned}
\Delta M \equiv & M(u, \xi)-M(\phi, 0) \\
= & \int_{-\infty}^{+\infty}\left[h^{\prime 2}+(\mathcal{C}-\phi) h^{2}+\sum_{i=0}^{\infty} \varphi_{i}^{\prime 2}\right. \\
& \left.+\left(\mathcal{C}-\frac{1}{2} \phi\right) \sum_{i} \varphi_{i}^{2}-\left(\frac{1}{3} h^{3}-\frac{1}{2} \sum_{i=0}^{\infty} \varphi_{i}^{2} h\right)\right] d x,
\end{aligned}
$$

where we have used $\mathcal{C}(V(u, \xi)-V(\phi, 0))=0$ and that $\phi$ is the soliton solution of $\mathrm{KdV}$ equation, and hence it satisfies

$$
\phi^{\prime \prime}+\frac{1}{2} \phi^{2}=\mathcal{C} \phi
$$

We denote $\delta^{3} M \equiv \int_{-\infty}^{+\infty}\left(-\frac{1}{3} h^{3}+\frac{1}{2} \sum_{i=0}^{\infty} \varphi_{i}^{2} h\right) d x$. We then have

$$
\left|\delta^{3} M\right| \leq \frac{1}{2} \sup |h|\|(h, \xi)\|_{H_{1}}^{2} \leq \frac{1}{2 \sqrt{2}}\|(h, \xi)\|_{H_{1}}^{3},
$$

where we have used $\sup |h| \leq \frac{\sqrt{2}}{3}\|h\|_{H_{1}}$.

Coming back to $\Delta M$, we get

$$
\begin{aligned}
|\Delta M| & \leq \int_{-\infty}^{+\infty}\left(h^{\prime 2}+\mathcal{C} h^{2}+\sum_{i=0}^{\infty} \varphi_{i}^{\prime 2}+\mathcal{C} \sum_{i=0}^{\infty} \varphi_{i}^{2}\right) d x+\left|\delta^{3} M\right| \\
& \leq \max (1, \mathcal{C})\|(h, \xi)\|_{H_{1}}^{2}+\frac{1}{3 \sqrt{2}}\|(h, \xi)\|_{H_{1}}^{3} .
\end{aligned}
$$


At $t=0$, we will assume that

$$
d_{I}[(u, \xi),(\phi, 0)]=\|(h, \xi)\|_{H_{1}}<\delta
$$

hence

$$
|\Delta M| \leq\left[\max (1, \mathcal{C})+\frac{1}{3 \sqrt{2}} \delta\right]\|(h, \xi)\|_{H_{1}}^{2} .
$$

$\Delta M$ is a conserved quantity on the space of solutions of system (5). By taking $\delta$ small enough in (23), we can make $\Delta M$ as small as we wish. The second step in the proof of stability is to argue that at any $t \geq 0, \Delta M$ satisfies the bound

$$
\Delta M \geq K d_{I I}[(u, \xi),(\phi, 0)]
$$

This bound completes the proof, in fact, we can make, at $t=0,|\Delta M|$ as small as we wish, and hence, we can always satisfy (14) for any given $\epsilon$.

We decompose $\Delta M$ into

$$
\Delta M=\delta_{h}^{2} M+\delta_{\xi}^{2} M+\delta^{3} M
$$

where

$$
\begin{aligned}
& \delta_{h}^{2} M=\int_{-\infty}^{+\infty}\left[{h^{\prime}}^{2}+(\mathcal{C}-\phi) h^{2}\right] d x \\
& \delta_{\xi}^{2} M=\int_{-\infty}^{+\infty}\left[\sum_{i=0}^{\infty} \varphi_{i}^{\prime 2}+\left(\mathcal{C}-\frac{1}{2} \phi\right) \sum_{i=0}^{\infty} \varphi_{i}^{2}\right] d x
\end{aligned}
$$

Using the same argument as in $[5,6]$, we obtain

$$
\delta_{h}^{2} M \geq \frac{1}{4} \int_{-\infty}^{+\infty}\left(h^{\prime 2}+\mathcal{C} h^{2}\right) d x-\frac{2}{5} \mathcal{C}^{\frac{1}{4}}\|(h, \xi)\|_{H_{1}}^{3}
$$

and

$$
\|h\|_{H_{1}} \geq d_{I I}(u, h)
$$

We will now obtain a lower bound for $\delta_{\xi}^{2} M$.

We consider the operator $H=-\frac{d^{2}}{d x^{2}}-\frac{1}{2} \phi$ with domain in the Hilbert space $L_{2}(\mathbb{R})$. It is a symmetric essentially self-adjoint operator. We denote with the same letter $H$ its selfadjoint extension. It has two eigenvalues $\lambda_{1}=-\mathcal{C}$ and $\lambda_{2}=-\frac{\mathcal{C}}{4}$ and a continuous spectrum $[0, \infty)$. The eigenfunctions are proportional to $\frac{1}{\cosh ^{2} z}$ and $\frac{\sinh z}{\cosh ^{2} z}$, respectively, where $z=\frac{1}{2} \mathcal{C}^{\frac{1}{2}}$. The spectral theorem for self-adjoint operators ensures the existence of a unitary transformation from the domain $D(H)$ in the Hilbert space $\mathcal{H}$ to $L_{2}(\mathbb{R}, d \rho)$. In the case in which $\mathcal{H}=L_{2}(\mathbb{R})$, this unitary transformation may be realized in terms of the eigenfunctions $\psi_{1}, \psi_{2} \in L_{2}(\mathbb{R})$ and the hypergeometric functions $\psi_{\lambda}(x)$ which satisfy point to point

$$
H \psi_{\lambda}=\lambda \psi_{\lambda}
$$


for $\lambda>0$, but do not belong to $L_{2}(\mathbb{R})$. Under the unitary map $f(x) \in L_{2}(\mathbb{R})$ can be expressed

$$
f(x)=F_{1} \psi_{1}(x)+F_{2} \psi_{2}(x)+\int_{0}^{\infty} F(\lambda) \psi_{\lambda}(x) d \rho(\lambda)
$$

where $F(\lambda)$ belongs to $L_{2}\left(\mathbb{R}^{+}, d \rho\right)$. The action of $H$ in $\mathcal{H}$ corresponds to the multiplication by $\lambda$ in $L_{2}\left(\mathbb{R}^{+}, d \rho\right)$ :

$$
H f=\lambda_{1} F_{1} \psi_{1}+\lambda_{2} F_{2} \psi_{2}+\int_{0}^{\infty} \lambda F(\lambda) \psi_{\lambda} d \rho(\lambda)
$$

We notice that for $f \in D(H)$, the third term on the right-hand member belongs to $L_{2}(\mathbb{R})$. $\psi_{\lambda}$ are normalized in order to have $\|f\|_{\mathcal{H}}^{2}=F_{1}^{2}+F_{2}^{2}+\int_{0}^{\infty} F^{2}(\lambda) d \rho(\lambda) . \psi_{1}, \psi_{2}, \psi_{\lambda}$ are pairwise orthogonal with the internal product in $L_{2}(\mathbb{R})$.

If we denote $g(x)=\int_{0}^{\infty} F(\lambda) \psi_{\lambda}(x) d \rho(\lambda), g \in L_{2}(\mathbb{R})$, it follows that

$$
\int_{-\infty}^{+\infty} H g d x=\int_{-\infty}^{+\infty}\left[\int_{0}^{\infty} \lambda F(\lambda) \psi_{\lambda} d \rho(\lambda)\right] d x
$$

its left-hand member is zero, hence

$$
\int_{-\infty}^{+\infty}\left[\int_{0}^{\infty} \lambda F(\lambda) \psi_{\lambda} d \rho(\lambda)\right] d x=0
$$

for any $F(\lambda)$ on the space $L_{2}\left(\mathbb{R}^{+}, d \rho\right)$.

We consider first $F(\lambda)$ to have support in the complement of a neighborhood of $\lambda=0$. We may then consider $\lambda F(\lambda)=\chi(\lambda)$, where $\chi(\lambda)$ is the characteristic function with value one in the interval $(\hat{\lambda}-\epsilon, \hat{\lambda}+\epsilon)$ and 0 otherwise. We have

$$
\int_{-\infty}^{\infty} \int_{\hat{\lambda}-\epsilon}^{\hat{\lambda}+\epsilon} \psi_{\lambda} d \rho(\lambda) d x=0
$$

for any $\hat{\lambda}$ in the support of $F(\lambda)$.

It then follows, by decomposing $F(\lambda)$, that

$$
\int_{-\infty}^{+\infty}\left[\int_{0}^{\infty} F(\lambda) \psi_{\lambda} d \rho(\lambda)\right] d x=0
$$

Since the $F(\lambda)$ considered is dense in $L_{2}\left(\mathbb{R}^{+}, d \rho\right)$, we conclude that (31) is valid for any $F(\lambda) \in L_{2}\left(\mathbb{R}^{+}, d \rho\right)$.

We may always decompose $\xi \in L_{2}(\mathbb{R})$ as

$$
\xi=F_{1} \psi_{1}+F_{2} \psi_{2}+\int_{0}^{\infty} F(\lambda) \psi_{\lambda} d \rho(\lambda)
$$

and use (18) together with (31) to obtain

$$
F_{1}=0 .
$$


We now consider the Rayleigh quotient of any $\xi$ in the domain of $H$

$$
R(\xi) \equiv \frac{\langle-\xi, H \xi\rangle}{\langle-\xi, \xi\rangle}
$$

$\langle$,$\rangle denotes the internal product in the L_{2}(\mathbb{R})$ space.

The eigenvalues satisfy (using the min-max theorem)

$$
\begin{aligned}
& \lambda_{1}=\inf \{R(\xi): \xi \in \operatorname{domain} H\}, \\
& \lambda_{2}=\inf \left\{R(\xi): \xi \perp \operatorname{span}\left(\psi_{1}\right)\right\} .
\end{aligned}
$$

We thus get

$$
\sum_{i}\left\langle\varphi_{i}, H \varphi_{i}\right\rangle \geq \lambda_{2} \sum_{i}\left\langle\varphi_{i}, \varphi_{i}\right\rangle
$$

From (27), and using $\lambda_{2}=-\frac{\mathcal{C}}{4}$, we obtain

$$
\delta_{\xi}^{2} M \geq \frac{3}{4} \mathcal{C} \sum_{i}\left\langle\varphi_{i}, \varphi_{i}\right\rangle
$$

and introducing a parameter $0 \leq \beta \leq 1$

$$
\begin{aligned}
\delta_{\xi}^{2} M= & (1-\beta) \delta_{\varphi}^{2} M+\beta \delta_{\varphi}^{2} M \\
\geq & (1-\beta) \sum_{i=0}^{\infty} \int_{-\infty}^{+\infty}\left(\varphi_{i}^{\prime 2}+\mathcal{C} \varphi_{i}^{2}\right) d x \\
& +(1-\beta) \sum_{i=0}^{\infty} \int_{-\infty}^{+\infty}\left(-\frac{1}{2} \phi\right) \varphi_{i}^{2} d x+\frac{3}{4} \beta \mathcal{C} \sum_{i=0}^{\infty} \varphi_{i}^{2} .
\end{aligned}
$$

Consequently, using $\phi \leq 3 \mathcal{C}$, we have

$$
\delta_{\xi}^{2} M \geq(1-\beta) \min (1, \mathcal{C})\|\xi\|_{H_{1}}^{2}
$$

for any $\beta$ satisfying $\frac{2}{3}<\beta<1$. In particular, for $\beta=\frac{3}{4}$,

$$
\delta_{\xi}^{2} M \geq \frac{1}{4} \min (1, \mathcal{C})\|\xi\|_{H_{1}}^{2} .
$$

From (28) and (33), we get

$$
\delta_{h}^{2} M+\delta_{\xi}^{2} M \geq \frac{1}{4} \min (1, \mathcal{C})\|(h, \xi)\|_{H_{1}}^{2}-\frac{2}{5} \mathcal{C}^{\frac{1}{4}}\|(h, \xi)\|_{H_{1}}^{2},
$$

and from (21),

$$
\delta^{3} M \geq-\frac{1}{2 \sqrt{2}}\|(h, \xi)\|_{H_{1}}^{2} .
$$


Finally,

$$
\Delta M \geq \frac{1}{4} l\|(h, \xi)\|_{H_{1}}^{2}-b\|(h, \xi)\|_{H_{1}}^{3},
$$

where

$$
l=\min (1, \mathcal{C}), \quad b=\frac{1}{2 \sqrt{2}}+\frac{2}{5} \mathcal{C}^{\frac{1}{4}} .
$$

We now use an argument in $[5,6]$ to show, for $\delta$ small enough, that

$$
\Delta M \geq \frac{1}{6} l\|(h, \xi)\|_{H_{1}}^{2} .
$$

Since

$$
\|(h, \xi)\|_{H_{1}} \geq d_{I I}[(u, \xi),(\phi, 0)]
$$

we then obtain

$$
\Delta M \geq \frac{1}{6} l\left\{d_{I I}[(u, \xi),(\phi, 0)]\right\}^{2}
$$

for $\delta$ small enough.

The stability proof has then been completed.

We may now relax the assumptions (17) and (18). The assumption (17) may be removed by an application of the triangle inequality as is [5]. While (18) may be relaxed by considering

$$
\tilde{\xi}=\xi-F_{1} \psi_{1}
$$

which satisfies $\int_{-\infty}^{\infty} \tilde{\xi} d x=0$.

Using again the triangle inequality for the distance $d_{I I}$, we get

$$
d_{I I}[(u, \xi),(\phi, 0)] \leq d_{I I}[(u, \tilde{\xi}),(\phi, 0)]+d_{I I}[(u, \xi),(u, \tilde{\xi})]
$$

where $d_{I I}[(u, \xi),(u, \tilde{\xi})]=\left\|F_{1} \psi_{1}\right\|_{H_{1}}$ is conserved by $\hat{H}_{\frac{1}{2}}$ and bounded by $\|(u, \xi)\|_{H_{1}}$ at $t=0$. The solitonic solution $(\phi, 0)$ is then stable in the sense (13), (14).

\section{Conclusions}

Following [1], we considered the breaking of the supersymmetry in the $N=1$ Super KdV system and analyzed a solitonic model in terms of a Clifford algebra-valued field. We showed the stability in the Liapunov extended sense of the ground state and the onesoliton solution of the integrable model. The approach introduced in [5] to prove the stability of the solitonic solutions of KdV equation and extended here for Clifford-valued fields may also be used in the stability analysis of supersymmetric solitons in the bosonization scheme in $[14,15]$.

Independently of the original motivation, the breaking of supersymmetry, the system we analyzed in this paper is interesting, because it contains the same soliton solutions as 
the $\mathrm{KdV}$ equation but is more realistic in the sense that the symmetry associated to the infinite number of conserved charges of $\mathrm{KdV}$ is broken.

\section{Competing interests}

The authors declare that they have no competing interests.

\section{Authors' contributions}

Both authors contributed equally and read and approved the final manuscript.

\section{Author details}

${ }^{1}$ Departamento de Física, Universidad de Antofagasta, Antofagasta, Chile. ${ }^{2}$ Departamento de Física, Universidad Simón Bolívar, Caracas, Venezuela. ${ }^{3}$ Departamento de Matemáticas, Universidad de Antofagasta, Antofagasta, Chile.

\section{Acknowledgements}

AR and AS are partially supported by Project Fondecyt 1121103, Chile.

Received: 3 July 2013 Accepted: 17 September 2013 Published: 07 Nov 2013

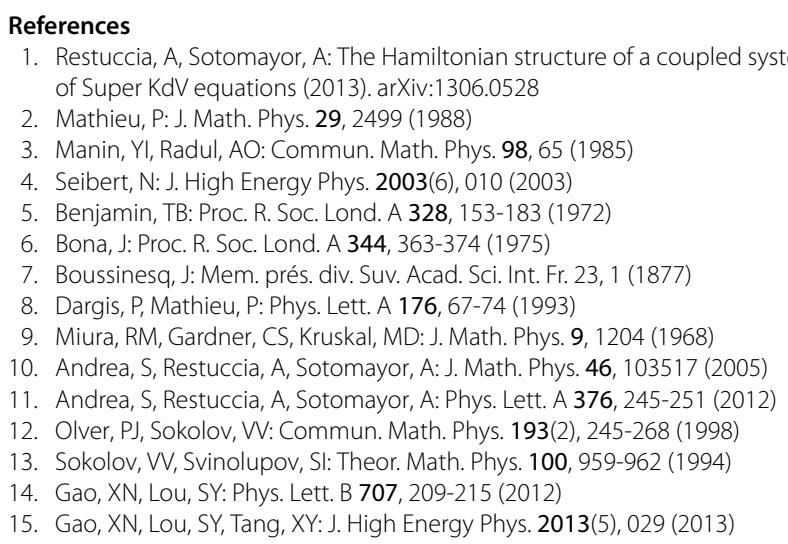

10.1186/1687-2770-2013-224

Cite this article as: Restuccia and Sotomayor: Stability of solitonic solutions of Super KdV equations under susy breaking conditions. Boundary Value Problems 2013, 2013:224

\section{Submit your manuscript to a SpringerOpen ${ }^{\circ}$ journal and benefit from:}

- Convenient online submission

Rigorous peer review

- Immediate publication on acceptance

- Open access: articles freely available online

- High visibility within the field

- Retaining the copyright to your article 